\title{
Revisiting Sub-Optimality of the Decentralized Decision Making: A Note
}

\author{
Amaresh Das \\ College of Business \\ Southern University at New Orleans \\ $\&$ \\ Department of Mathematics \\ University of New Orleans \\ Tel: 504-393-7849Ｅ-mail: adas2@cox.net
}

\begin{abstract}
The famous 1968 Witsenhausen counterexample demonstrated that for a multi-agent linear quadratic Gaussian (LQG), the optimal linear solution is, in general sub-optimal. The counterexample was a chosen distributed control system (and hence a system with a non-classical information pattern) that was otherwise quadratic and Gaussian. For this system, Witsenhausen provided a nonlinear control law that outperformed the optimal linear control law and demonstrated that a measurable optimal control law should exist. The note gives an example, which is easier and more sophisticated than the Witsenhausen example but still allows for a convex formulation over a set of complicated constraints.
\end{abstract}

Keywords: Linear quadratic Gaussian (LQG), Kalman filter, Borel function 


\section{Introduction}

A team decision problem consists of a group of decision makers seeking to maximize a common objective that depends on the group's joint decision. The difficulty associated with a team decision problem stems from the fact that each decision maker is making a decision independently in response to incomplete information. Decision makers are allowed to communicate their information to one another within a given information structure; however, such actions bear communication costs. The goal of the team decision problem is to find the optimal policy for the decision makers and the optimal information structure such as to minimize a cost function that incorporates the original objective, the available information, and the communication costs. One example of a team decision problem that has received a significant degree of research attention is the Witsenhausen counterexample (WC) (1968).

The counterexample has inspired a large volume of engineering researches along three related themes. The first body of work is devoted to finding the elusive optimal control law for the problem. For the simplicity with which the problem is stated, it is interesting to note that the optimal control law is still unknown. The second theme is in refining the classification of distributed LQG systems into those for which affine laws are optimal, and those for which affine laws are not optimal. The authors consider a parametrized family of two stage stochastic control problems. The family includes the Witsenhausen counterexample. Bansal and Basar (1987) use results from information theory to arrive at the optimality result. The authors show that affine controls are still optimal for a deterministic variant of the Witsenhausen counterexample if the cost function is the induced two-norm instead of the expected two-norm in the stochastic variant. The third theme has been in viewing the counterexample as a bridge between control and communication. Mitter and Sahai (1999) observe that the original Witsenhausen problem is in essence a communication problem between the two controllers. They back up this observation by proposing control strategies that are explicitly based on quantization of the initial state.

As we can see, since Witsenhausen put forward his remarkable counterexample, there have been many attempts to develop efficient methods for solving the functional optimization problem that is non-convex (Note1). The WC is illustrated in Figure 1 and has the following elements:

_External Signals: $\quad x$; v; independent random variables with finite second moments. We assume independent Gaussian random variables, where $x \sim N\left(0 ; \sigma^{2}\right) ; v \sim N(0 ; 1)$.

- Information (Observation): $x ; y \quad$ where $y=u_{1}+v$ :

- Decision Variables: $u_{1}=f(x) ; \quad u_{2}=g(y)$; where $(f ; g)$ is any pair of Borel functions. - Cost objective: 
Min $J=E\left[\begin{array}{lll}k^{2} & \left.\left(u_{1}-x\right)^{2}+\left(u_{2}-u_{1}\right)^{2}\right]\end{array}\right.$

(1)

$$
f, g
$$

The goal is for decision maker, DM1 one to estimate the external signal $x$ and DM2 to estimate $u_{1}$ which is corrupted by the noise signal $v$. Although the WC involves only two decision makers, it possesses almost all of the main difficulties inherent in any decentralized team decision problem.

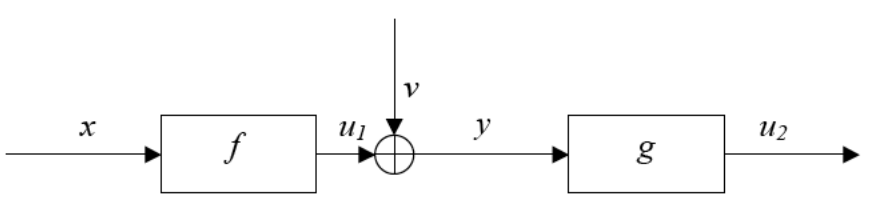

Fig. 1. Information structure of the Witsenhausen Counterexample

In a decentralized decision making, if the flow if information to a central optimizer is less than complete then one has, in fact, a situation with many decision makers, who do not completely share information even if they share a common goal. Decentralization often leads effectively to a type of multi-agent situation in which the definition of the 'players' and the 'rules of the game' are treated with some success in a dynamic (i.e. recursive) fashion (Note 2). The WC, therefore, is essentially a team decision problem but with differing information (see Marachank and Radner (1972) Ho and Chu (1972), Radner (1991).

The purpose of this note is to illustrate by way of an example that some of the most familiar features of the single-agent case do not survive a multi-agent quadratic Gaussian (LQG) problem (Note 3) and the optimum linear solution is, in general, sub-optimal. Here we shall give an example which is easier but more sophisticated than the Witsenhausen's. We achieve a better solution than the one previously known. Moreover, we show that the learning approaches are simple and automated and they are easy to extend for solving general functional optimization problems. Our purpose is not to emphasize the connections between control and information theory nor our purpose is to captures the essence of the implicit interactions that are possible in distributed control systems (Note 4).

\section{A Simple LQG Problem and the Optimal Linear Solution}

Assume the scalar state equation

$$
\mathrm{X}_{\mathrm{t}+1}=\mathrm{AX}_{\mathrm{t}}+\sum_{\mathrm{j}=1}^{\mathrm{r}} \mathrm{u}_{\mathrm{jt}}+\varepsilon_{\mathrm{t}}
$$


where $u_{j t}$ is the control exerted by the jth agent, based upon observation of $y_{j s}=X_{s}+\eta_{j s}(s \leq t)$. The process $\left\{\varepsilon_{t}\right\}$ and $\left(\eta_{j t}\right)(j=1,2, \ldots r)$ are supposed independent white noise processes, with $\operatorname{var}\left(\varepsilon_{t}\right)=N$, $\operatorname{var}\left(\eta_{j t}\right)=M$. We assume equilibrium behavior and the common criteria function

$$
\mathrm{C}=\mathrm{RX}^{2}+\mathrm{Q} \sum_{\mathrm{j}} \mathrm{u}_{\mathrm{j}}^{2}
$$

whose expectation is to be minimized in the stationary state. This is then a scalar regulation problem with many controllers.

In view of the symmetry of the problem over agents, it seems likely (although the point should be proved) that the optimal controls should take a common form

$$
\mathrm{u}_{\mathrm{jt}}=\beta(\mathrm{T}) \mathrm{y}_{\mathrm{jt}}
$$

where $\beta(T)$ is a realizable operator

$$
\beta(T)=\sum_{0}^{\infty} \beta_{\mathrm{s}} \mathrm{T}^{\mathrm{S}}
$$

Using the techniques given in Whittle and Rudge (1979) one finds the evaluation

$$
\mathrm{EC}=\dot{\mathrm{A}}\left[| \theta | ^ { 2 } ( \mathrm { R } + \mathrm { rQ } | \beta | ^ { 2 } ) \left(\mathrm{N}+\mathrm{rMQ}|\beta|^{2}\left(1+\mathrm{z} \beta \theta+\mathrm{z}^{-1} \boldsymbol{\beta}^{-}\right.\right.\right.
$$

where $r$ is the number of agents and

$$
\theta=[1-\mathrm{Az}-\mathrm{rz} \beta(\mathrm{z})]^{-1}
$$

In (6) we follow the conventions $\beta=\beta(\mathrm{z}), \bar{\beta}\left(\mathrm{z}^{-1}\right),|\beta|^{2}=\beta \bar{\beta}$ etc., and the operator extracts the absolute term in the expansion on $|z|=1$. It is convenient to define $D, \alpha$ by

$$
\mathrm{D}(\mathrm{z})=\theta(\mathrm{z})^{-1}=\alpha(\mathrm{z})-\mathrm{rz} \beta(\mathrm{z})
$$

Theorem 
The optimal linear operator $\beta$, given in terms of $D=\theta^{-1}$ by (7), is determined by the fact that $\mathrm{D}=\sum_{\mathrm{s}=0}^{\infty} \mathrm{d}_{\mathrm{s}} \mathrm{z}^{\mathrm{s}} \quad$ is the canonical factor of

$$
|\mathrm{D}(\mathrm{z})|^{2}=\frac{(4 \lambda \mu+\mathrm{v})^{1 / 2}-\mathrm{v}}{2 \mu}
$$

where

$$
\begin{gathered}
\lambda(\mathrm{z})=\left(\mathrm{rR}+\mathrm{rQ}|\alpha|^{2}\right)\left(\mathrm{rN}+\mathrm{M}|\alpha|^{2}\right) \\
\mu=\mathrm{QM}(\mathrm{r}-1) \\
\mathrm{v}(\mathrm{z})=\mathrm{v}_{0}+\mathrm{QMA}(\mathrm{r}-\mathrm{z})\left(\mathrm{z}+\mathrm{z}^{-1}\right.
\end{gathered}
$$

and $\mathrm{v}_{0}$ is a scalar to be determined by the condition $\mathrm{d}_{0}=1$. If $\mathrm{NR}>0$ then the solution for $\beta$ (z) implied by (6) is rational and if and only if $\mu=0$

Proof

One finds with some reduction that relation (4) can be rewritten

$$
\begin{aligned}
\mathrm{r}^{2} \mathrm{EC}=\dot{\mathrm{A}}[ & \frac{\lambda(\mathrm{z})}{|\mathrm{D}|^{2}}+(\mathrm{r}-1) \mathrm{QM}|\alpha-\mathrm{D}|^{2} \\
& +\left(\mathrm{rRM}+\mathrm{rQN}+\mathrm{QM}|\alpha|^{2}\right)\left(1-\frac{\alpha}{\mathrm{D}}-\frac{\bar{\alpha}}{\mathrm{D}}\right]
\end{aligned}
$$

Minimization of this expression with respect to $\beta$ is equivalent to minimization with respect to $\mathrm{d}_{1}, \mathrm{~d}_{2} \ldots .$, and leads to the relation

$$
\lambda|\mathrm{D}|^{-2}-\mu|\mathrm{D}|^{2}-\mathrm{v}=\sum_{-\infty}^{0}
$$

where the sum represents a series in non-positive powers, convergent on the unit circle. The coefficients of $z^{s}$ and $z^{-s}$ are equal in the expansion of the left-hand side of (9), so that 
the right-hand side must reduce to a constant. This constant can be absorbed into $\mathrm{v}_{0}$ and therefore, taken as zero. Solving the resulting quadratic equation for $|\mathrm{D}|^{2}$ and taking the solution, which is positive on the unit, circle, we deduce (8). Since $\theta=\mathrm{D}^{-1}$ is to be both realizable and stable, we require $\mathrm{D}$ to be the canonical factor of expression (8).

\section{Remarks}

The significance of rationality of $\beta(z)$ is that it would indicate that the optimal control (3) could be generated by a finite number of simple recursive relations. This was true for the Kalman filter: the state estimate $\mathrm{X}$ was generated by a first-order recursion and this then supplied the optimal control $\mathrm{u}=\mathrm{K} \hat{\mathrm{X}}$. However, for the multi-agent case there is generally no way of seeing things in a finitely recursive manner. One can explain this in terms of the need for agents to try and understand what other agents are observing and doing. So, one has not only to construct one's estimate of state, but also one's own estimate of other's estimates, one's estimates of other's estimates of estimates., etc. This infinite regress implies infinite-order recursions.

One returns to finiteness in the case QMR $(r-1)=0$. i. e, when either $r=1$ ( the single-agent case) or when $M=0$ ( communality of information means that one is effectively back to the single-agent case) or when $\mathrm{Q}=0$ ( and, control being unpenalized, agents try to reduce the state variable to zero at every stage).

\section{Conclusion}

The WC is a simple example of a linear-quadratic-Gaussian team problem. Before Witsenhausen put forth this counterexample, it was conjectured that in any LQG team problem the optimal controllers are linear. Witsenhausen proved that the WC has an optimal solution that is not of the linear type. Thus, he claimed that the conjecture is not necessarily true if the information pattern is not classical, i.e., the information available in earlier decision makers is not available to latter decision makers. Since then, many researchers have focused on understanding the role of information structures in team decision problems and on developing more efficient methods to find improved solutions for the WC. The work in this paper belongs to the second kind. This note gives an example, which is much easier, but more sophisticated than the Witsenhausen example but still allows us for a convex formulation of the problem over a set of complicated constraints.

\section{References}

Bagchi, A and Basar, T (1980) "Team Decision Theory for Linear Continuous-time Systems", IEEE Transactions on Automatic Control, 6, 1154-1161. 
Baglietto M, Parisini T and Zoppoli R (2001), "Numerical Solutions to the Witsenhausen Counterexample by Approximating Networks," IEEE Transactions on Automatica Control, 46, 9, 1471-1477.

Bansal , R and T. Basar (1987) "Stochastic teams with nonclassical information revisited: When is an affine control optimal?" IEEE Transactions on Automatic Control, 24, 4, 76-87.

Ho, Y. C and Chu, K C (1972). "Team Decision Theory and Information Structures in an Optimal Control Problems, IEEE, Transaction in Automatic Control, AC 17, 15-28.

J. Marschak (1995), “Elements for a Theory of Teams,” Management Science, 1, 2, 127-137.

Marschank J and Radner, R (1986). Economic Theory of Teams, Yale University Press.

S. K. Mitter and A. Sahai, (1999). "Information and control: Witsenhausen revisited," in Learning,Control and Hybrid Systems: Lecture Notes in Control and Information Sciences 241, Y. Yamamoto and S. Hara, Eds. New York, NY: Springer, 1999, 281- 293.

$\mathrm{Na}$ Li, Marden Jason, Shamma Jeff. (2009). "Learning Approaches to the Witsenhausen Counterexample from the Pont of View Potential Games", 48IEEE Conference on Decision and Control. 135-142

M. Rotkowitz (2006). "Linear Controllers are Uniformly Optimal for the Witsenhausen Counterexample," IEEE Conference on Decision and Control. 3, 2, 43-78

M. Rotkowitz (2006). "A Characterization of Convex Problems in Decentralized Control," IEEE Transactions on Automatic Control, 51, 2, 1215-1220.

H. P. Young. (2004). Strategic Learning and Its Limits, Oxford University Press, Oxford, UK.

Whittle, P. and Rudge J P. (1977). " The Optimal Linear Solution of a Systematic Team Control Problem, Journal of Applied Probability, 11, 377-381.

H. S. Witsenhausen. (1968). "A Counterexample in Stochastic Optimum Control," SIAM J. Control, 6, 1, 131-147.

\section{Notes}

Note 1. Witsenhausen proved that the optimal controller for the WC have these properties. (i) if $\int$ is optimal then $\mathrm{E}\left[\int(\mathrm{x})\right]=0, \mathrm{E}\left[\int(\mathrm{x})^{2}\right] \leq 4 \sigma^{2}$ (ii) given a fixed $\int(\mathrm{x})$ having zero means and variance not exceeding $4 \sigma^{2}$, the optimal choice for $g($.$) is given by$

$$
\left.\mathrm{g}^{\bullet}(\mathrm{y})=\mathrm{E}\left[\int(\mathrm{x}) \mid \mathrm{y}\right]\right\rangle=\frac{\left.\mathrm{E}_{\mathrm{x}}\left[\int(\mathrm{x}) \phi(\mathrm{y})-\int(\mathrm{x})\right)\right]}{\mathrm{E}_{\mathrm{x}}\left(\phi\left(\mathrm{y}-\int(\mathrm{x})\right]\right.}
$$


where $\phi($.$) is the standard Gaussian density. The corresponding pay-off function$

is

$\mathrm{J}^{*}\left(\int\right)=\mathrm{J}\left(\int, \mathrm{g}_{\int}^{*}\right)=\mathrm{k}^{2} \mathrm{E}\left[\mathrm{x}-\int(\mathrm{x})\right\rceil^{2}+1-\int\left(\mathrm{D}_{\int}\right)$ where $\left.\mathrm{I}_{\left(\mathrm{D}_{j}\right)}\right)=\int\left[\frac{\mathrm{d}}{\mathrm{dy}} \mathrm{D}_{\int}(\mathrm{y})\right]^{2} \frac{\mathrm{dy}}{\mathrm{D}_{\int}(\mathrm{y})}$

which is called the 'Fisher Information' of the random variable y with density $\mathrm{D}_{f}$ (y) being

$\mathrm{D}_{f}(\mathrm{y})=\int \phi\left(\mathrm{y}-\int(\mathrm{x})\right) \phi\left(\mathrm{x} ; 0, \delta^{2}\right) \mathrm{dx}$

Note 2. Recently some have even started formulating the WC as a potential game and using the learning approach fading memory. One approach to formulating the WC as a potential game has been to model it as a game between the two decision makers where the actions available to each decision maker are the set of possible control laws. This approach leads to some challenges since the cardinality of each action set is infinite. Others, for example (Na, Marden and Shamma (2009), Bagchi and Basar (1980) formulate this WC as a potential game in an alternative fashion. He used $n$-step functions for $\mathrm{f}(\mathrm{x})$ and modeled each interval as a player and the value taken by each interval as the player's action. The number of players and the size of action sets were determined by the way of discretizing the problem.

Note 3. The WC is a simple example of a linear-quadratic-Gaussian (LQG) team problem [9]. Before Witsenhausen put forth this counterexample, it was conjectured that in any LQG team problem the optimal controllers are linear. Witsenhausen proved that for any $\mathrm{k}>0$, the WC has an optimal solution that is not necessarily of the linear type

Note 4. When engineers and physicists take an information-theoretic view, they get inspired by the "cognitive radio channel" and deterministic channel models that lead directly to an asymptotically infinite-dimensional version of the counterexample for which new upper and lower bounds reveal that it is always possible to achieve within a factor of two of the optimal cost. The results are then pulled back to the finite dimensional case using our "Platonic perspective" on sphere-packing bounds to get a similar factor of eight approximate optimality result for Witsenhausen's original scalar counterexample. 